\title{
Design of bi-material pixels of an uncooled optic readout IR FPA
}

by X. Zhang* and D. C. Zhang **

*Department of Photo-electronics, Communication University of China, Beijing 100024, China, zhangxia@cuc.edu.cn **Institute of Microelectronics, Peking University, Beijing 100871, China, Dchzhang@ime.pku.edu.cn

\section{Abstract}

The analysis and optimized design of an uncooled optic readout infrared FPA pixel composed of infrared absorption layer SiNx and bi-material beam surface layer Au (also as visible light reflection layer) based on MEMS technology are presented in the paper. By modeling and simulation, the thickness of SiNx, Au and adhesive metal layer, and the thickness matching relationship of these materials are verified, and an optimized bi-material beam of AulCrlSiNx $(0.29 \mu \mathrm{m} \backslash 5 \mathrm{~nm} \backslash 0.4 \mu \mathrm{m}, 104 \mu \mathrm{m}$ in equivalent beam length) in the pixel has thermo-mechanical response as high as $2.573 \times 10^{-3} \mathrm{rad} / \mathrm{K}$, and the visible light reflector in it remains flat enough.

\section{Introduction}

A thermal image system using an uncooled infrared focal plane array (IR FPA) as detector is a fast developing field, due to its small size, low power consumption, and low cost. The IR FPA based on Micro-Electro-Mechanical Systems (MEMS) technology is a key element of the system, directly affecting the thermal image quality. Although there are different types of the IR FPAs used for uncooled optic readout (UOR) thermal image systems, the pixels of the various UOR IR FPAs generally consist of bi-material reflectors reflecting visible light and bi-material beams driving the reflectors to rotate when the pixels are radiated by IR. Bi-material structures based on MEMS technology usually composed of a layer of silicon-based thin film with high IR absorptivity, low coefficient of thermal expansion (CTE) and stress, formed by chemical vapour deposition and a layer of metal thin film with high CTE and reflectivity of visible light, by physical vapour deposition. If the two-layer films are not able to form a firm structure, an adhesive layer is needed. While the pixel operates, it is hoped that reflectors remain flatter and the bi-material beams have higher thermomechanical response. In the paper, the optimized design of an UOR IR FPA pixel composed of SiNx as infrared absorption layer, Au as bi-material beams surface layer and visible light reflection layer, and an adhesive metal layer between SiNx and Au are presented, with the help of the analysis of the relationship between the thickness of materials used for bi-material beam and the thermo-mechanical response of the pixel, the thickness of IR radiation absorption layer and the visible light reflection layer.

\section{Films thickness and thermo-mechanical response}

A bi-material beam with an adhesive layer composed of material 1,2 and 3 is shown in Figure 1. $L$ and $b$ are the length and wide of the beam. $h_{1}, h_{2}$ and $h_{3}$ are the thickness of material 1,2 and $3 . E_{1}, E_{2}$ and $E_{3}$ are the elastic modulus of material 1, 2 and 3, respectively. $\alpha_{1}, \alpha_{2}$ and $\alpha_{3}$ are the CTE of material 1,2 and 3, and $\alpha_{1}<\alpha_{2}, \alpha_{3}$.

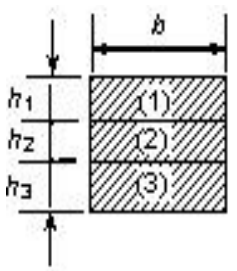

(a)

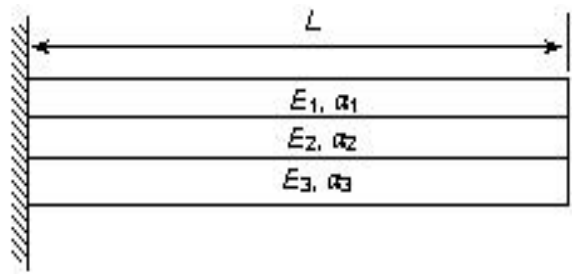

(b)

Fig. 1. Schematic diagram of bi-material beam

The temperature of the beam increases due to the beam absorbing incident IR flux, resulting in material 1,2 and 3 expanding. At the same time, material 1 will be extended by material 2 and material 2 will also be compressed by material 1 at the interface between materials; material 2 and material 3 will be extended or compressed by each other at the interface between materials. The mismatch of the CTEs of different materials will cause the beam bending. Based on thermal stress and combined deformation on mechanics of materials, a thermo-mechanics response model used to guide the selection of material and thickness, and the matching of the thickness of materials used for the pixel is written as [1]: 


$$
\frac{\Delta \theta}{\Delta T}=\frac{6 L\left\{\left[\left(h_{2}+h_{3}\right)+\left(h_{1}+h_{2}\right)\left(1+\frac{h_{2} E_{2}}{h_{3} E_{3}}\right)\right]\left(\alpha_{2}-\alpha_{1}\right)+\left[\left(h_{2}+h_{3}\right)\left(1+\frac{h_{2} E_{2}}{h_{1} E_{1}}\right)+\left(h_{1}+h_{2}\right)\right]\left(\alpha_{3}-\alpha_{2}\right)\right\}}{\left(E_{1} h_{1}^{3}+E_{2} h_{2}^{3}+E_{3} h_{3}^{3}\right)\left(\frac{h_{1} E_{1}+h_{2} E_{2}+h_{3} E_{3}}{h_{1} E_{1} h_{3} E_{3}}\right)+6\left(h_{1}+h_{2}\right)\left(h_{2}+h_{3}\right)+3\left[\left(h_{1}+h_{2}\right)^{2}\left(1+\frac{h_{2} E_{2}}{h_{3} E_{3}}\right)+\left(h_{2}+h_{3}\right)^{2}\left(1+\frac{h_{2} E_{2}}{h_{1} E_{1}}\right)\right]}
$$

Eq. (1) indicates that the value of $\Delta \theta / \Delta T$ of a bi-material beam, which is the ratio of the maximum bending angle $\Delta \theta$ of the free end of the beam shown as Figure 2 and the temperature change $\Delta T$, i. e., the thermo-mechanical response of the pixel, is proportional to the beam length $L$. In addition, the value of $\Delta \theta / \Delta T$ is related to the thickness and the elastic modulus of material 1, 2 and 3, and the CTE difference between two materials in contact with each other.

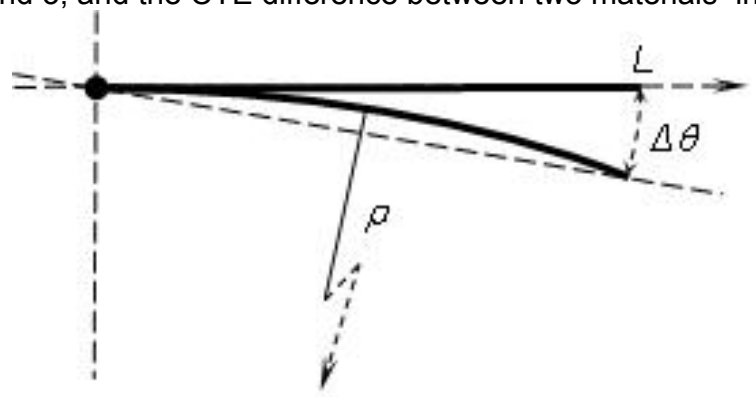

Fig.2. Bending angle of bi-material beam

\subsection{Surface and adhesive metal film thickness}

Taking consideration of materials property and process feasibility, Au or Al and low stress SiNx are commonly used for surface layer of the beam and reflector, and IR absorption layer of the pixel [2,3]. As the metal Al is able to react with $\mathrm{Si}, \mathrm{C}$ and $\mathrm{OH}^{-}$on the surface of silicon based materials, resulting that $\mathrm{Al}$ and SiNx firmly adhere together each other[4]. However, Au does not have this effect and has almost no reaction with SiNx at room temperature, resulting that the bi-material structure is not firm, an adhesive layer, like $\mathrm{Al}, \mathrm{Cr}$, $\mathrm{Ti}$ or $\mathrm{Pd}$, is needed, forming a sandwich structure with the surface metal layer and IR absorption layer together. The parameter of materials based on MEMS technique, is listed in table 1.

Table 1. Material parameters used for the bi-material structure $[5,6,7]$

\begin{tabular}{|l|l|l|l|l|l|l|l|}
\hline material & $\begin{array}{l}\text { Young's } \\
\text { modulus, } \\
\mathrm{E}\left(10^{9} \mathrm{~Pa}\right)\end{array}$ & $\begin{array}{l}\text { Expansion } \\
\text { coefficient, } \\
\alpha\left(10^{-6} /{ }^{\circ} \mathrm{C}\right)\end{array}$ & $\begin{array}{l}\text { Specific heat, } \\
\mathrm{c}(\mathrm{J} / \mathrm{kg} \cdot \mathrm{K})\end{array}$ & $\begin{array}{l}\text { IR absorptivity, } \\
\mathrm{a}(\%)\end{array}$ & $\begin{array}{l}\text { Reflectivity of } \\
\mathrm{IR}(10 \mu \mathrm{m}), \\
R(\%)\end{array}$ & $\begin{array}{l}\text { Density, } \\
\rho\left(10^{3} \mathrm{~kg} / \mathrm{m}^{3}\right)\end{array}$ & $\begin{array}{l}\text { Poisson's } \\
\text { ratios, } \mathrm{V}\end{array}$ \\
\hline $\mathrm{SiNx}$ & 180 & 0.8 & 690 & 92 & -- & 2.4 & 0.42 \\
\hline $\mathrm{Au}$ & 73 & 14.2 & 130 & 2 & 98.4 & 19.3 & 0.27 \\
\hline $\mathrm{Al}$ & 70 & 25.0 & 942 & 3 & 98.1 & 2.7 & 0.35 \\
\hline $\mathrm{Ti}$ & 106 & 8.5 & 577 & 10 & -- & 4.5 & 0.19 \\
\hline $\mathrm{Cr}$ & 279 & 8.5 & 450 & 15 & -- & 7.2 & 0.21 \\
\hline $\mathrm{Pd}$ & 180 & 12.4 & 240 & 3 & -- & 12.0 & 0.33 \\
\hline
\end{tabular}

The dimensions of the designed beam are $50 \mu \mathrm{m}$ in length $(L), 2 \mu \mathrm{m}$ in thickness $(h 1)$ of SiNx. Au is chosen as surface material. $\mathrm{Al}, \mathrm{Cr}$, Ti or Pd is used as an adhesive layer ( $h 2)$, respectively, where the CETs of $\mathrm{Cr}$, $\mathrm{Ti}$ and $\mathrm{Pd}$ are smaller than $\mathrm{Au}$, but the CET of $\mathrm{Al}$ is larger than $\mathrm{Au}$. According to eq. (1), applying the dimensions of the beam, and material parameters in table 1 , a group of $3 D$ curves is shown in figure $3,4,5$, and 6 . In the figures, two axes in horizontal plane represent the thickness of $\mathrm{Au}$ and $\mathrm{Al}, \mathrm{Au}$ and $\mathrm{Cr}, \mathrm{Au}$ and $\mathrm{Ti}$ or $\mathrm{Au}$ and $\mathrm{Pd}$ respectively; the vertical axis represents the value of $\Delta \theta / \Delta T$ of the free end of the beam.

The figures show clearly that the AulAIISiNx beam has a larger value of $\Delta \theta / \Delta T$ compared with AulCrlSiNx, AulTilSiNx and AulPdISiNx beams, when the film thickness of SiNx is fixed. 


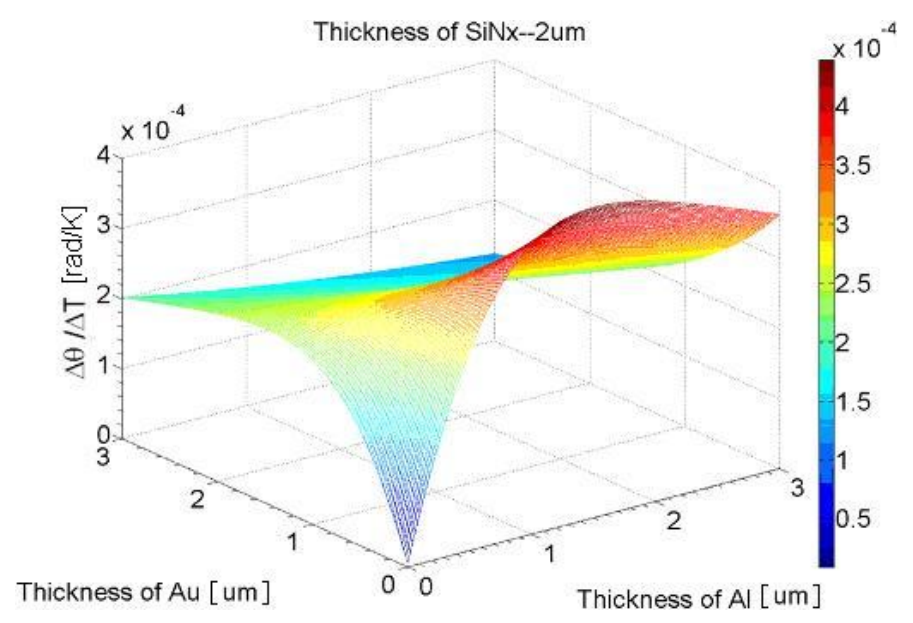

Fig. 3. Relationship between $\Delta \theta / \Delta T$ and Au-Al thickness

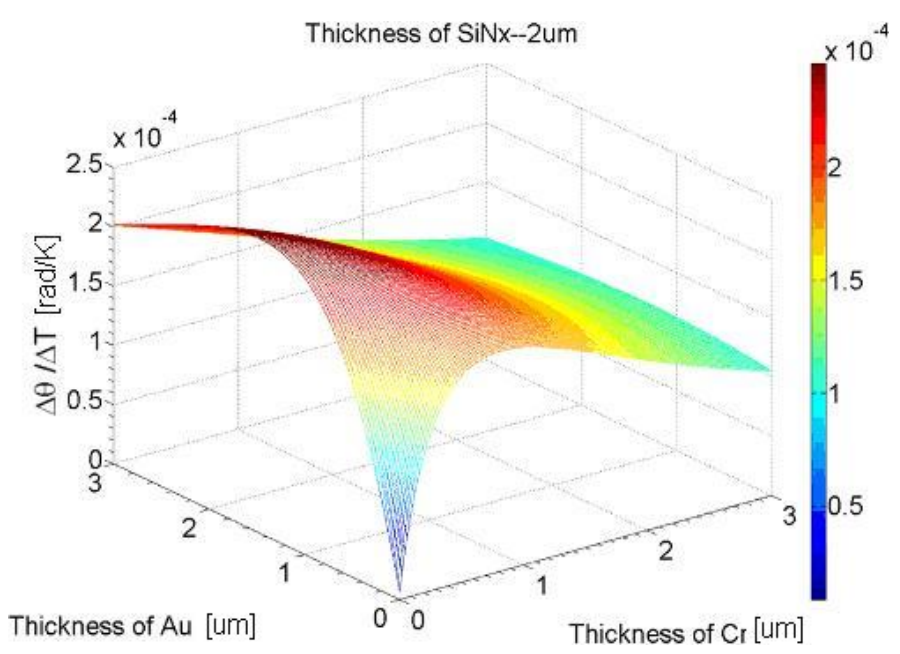

Fig. 4. Relationship between $\Delta \theta / \Delta T$ and $A u-C r$ thickness

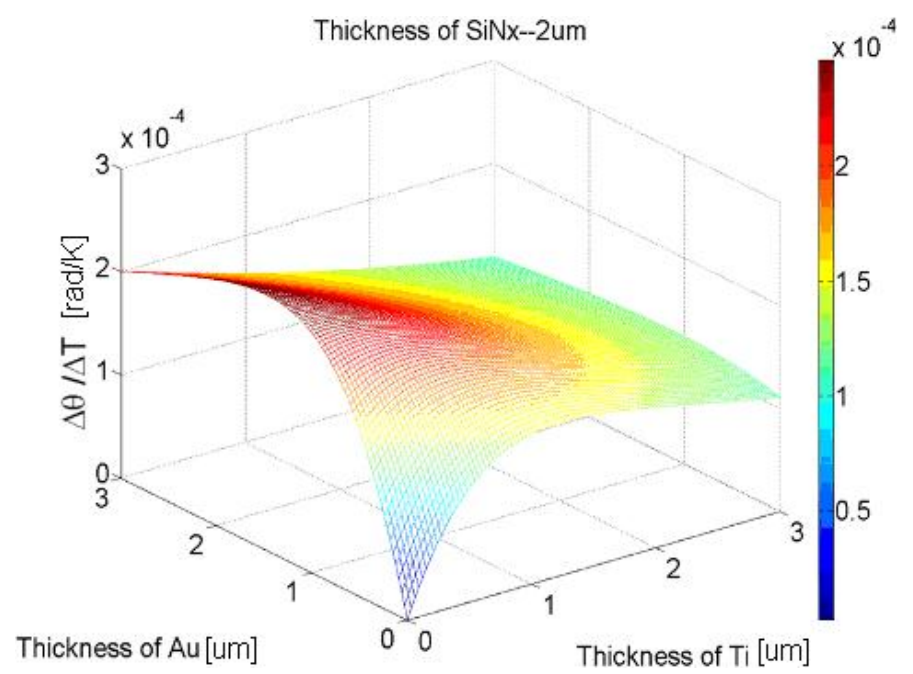

Fig. 5. Relationship between $\Delta \theta / \Delta T$ and Au-Ti thickness 


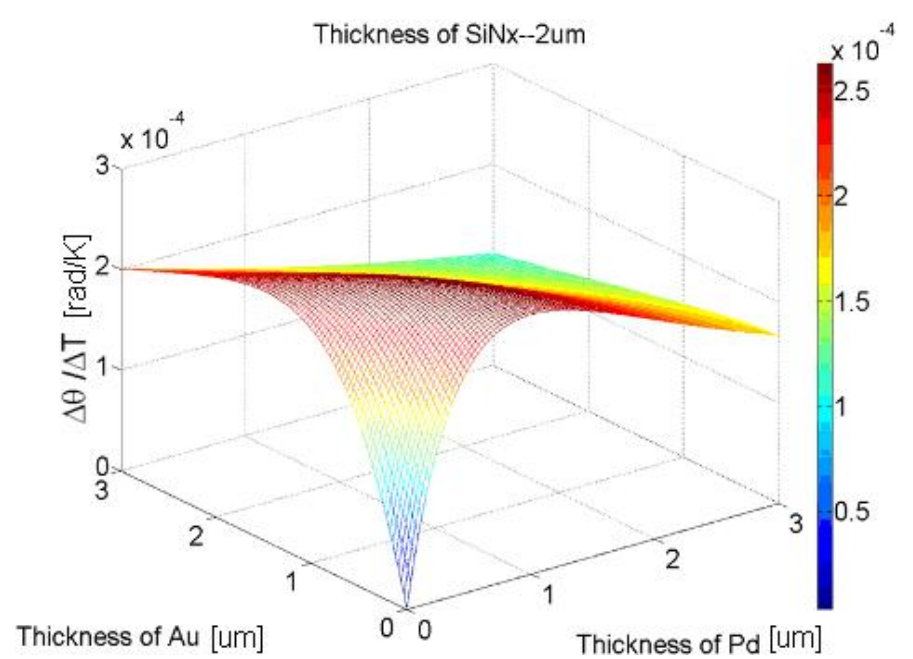

Fig. 6. Relationship between $\Delta \theta / \Delta T$ and Au-Pd thickness written as:

By analyzing, the conclusions about the thickness relationship between surface layer and adhesive layer can be

(1) If the difference between the CET of two metals is larger, regardless of the metal film with smaller CET lies in the middle or the surface layer, such as Au in figure 3 and $\mathrm{Cr}$, Ti in figure 4 and 5 , the thinner the metal film thickness, the larger the obtained value of $\Delta \theta / \Delta T$ of the beam will be.

(2) If the CET of two metals is closed to each other, such as Au and Pd in figure 6, a largest value of $\Delta \theta / \Delta T$ may be obtained while the thickness of two metal films is close to each other. For instance, the Au and Pd thicknesses are $0.66 \mu \mathrm{m}$ and $0.63 \mu \mathrm{m}$ respectively, resulting that the $\Delta \theta / \Delta T$ has a largest value of $2.6 \times 10^{-4} \mathrm{rad} / \mathrm{K}$.

The above conclusions (1) and (2) show that the value of $\Delta \theta / \Delta T$ depends on matching thickness of two metals on $\mathrm{SiNx}$. It is not true that the thinner the thickness of adhesive layer, the larger the value of $\Delta \theta / \Delta T$ will be. This result is important for the beam to obtain a high thermo-mechanical response.

(3) While two metal film thicknesses on SiNx approach zero, the value of $\Delta \theta / \Delta T$ is close to zero also. This structure is ideal for a reflector in the pixel. However, the too thin metal will be penetrated by the incident visible light [8]. Therefore, the metal thickness of the reflection surface should be larger than $40 \mathrm{~nm}$ in order to ensure sufficient reflectivity to visible light.

\subsection{Infrared absorption film thickness}

Taking an example of AulCrlSiNx structure, according to the conclusion (1) in section 2.1 that is the thinner the metal with smaller CET, the larger the obtained value of $\Delta \theta / \Delta T$ of the beam will be. Therefore, if thickness of $\mathrm{Cr}$ is designed as $5 \mathrm{~nm}$, the figure 5 is attained by eq. (1). The figure shows that there is non-linier relationship between the $\Delta \theta / \Delta T$ and thickness of $\mathrm{Al}$ and $\mathrm{SiNx}$ while the thickness of $\mathrm{Cr}$ is fixed; the thinner the thickness of SiNx and $\mathrm{Au}$, the larger the value of $\Delta \theta / \Delta T$ of the beam will be. This structure has a high thermo-mechanical response.

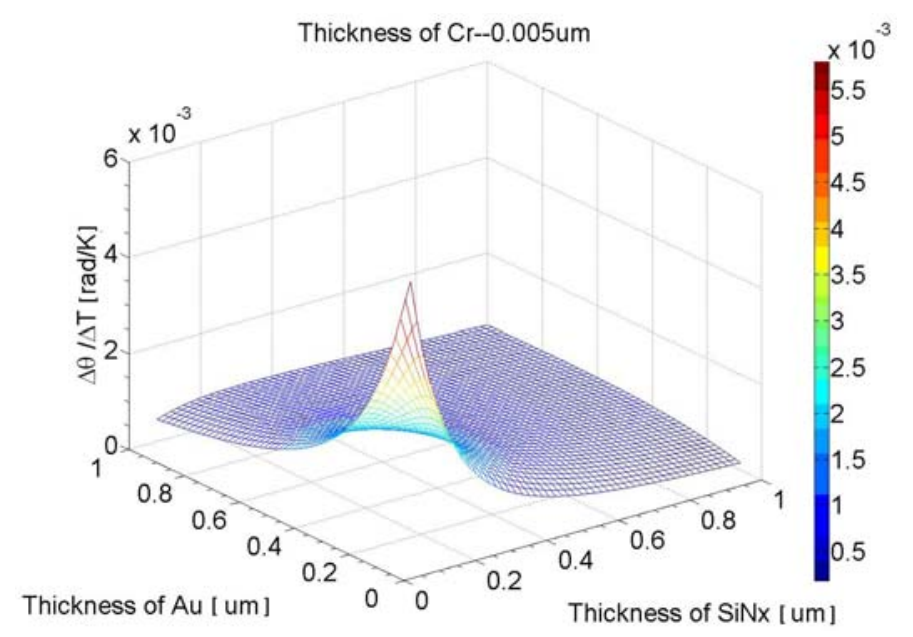

Fig. 7. Relationship between $\Delta \theta / \Delta T$ and Au-SiNx thickness 
Assuming that the intensity of incident IR radiation on the surface of IR absorption layer is $I_{0}$, the reflectivity of IR absorption material is R. According to P. Bouguer's law [9], when the incident depth is $x$, the IR intensity is expressed as

$$
I=I_{0}(1-R) \exp (-\alpha x)
$$

Where

$$
\alpha=\frac{4 \pi n \kappa}{\lambda}
$$

Where $\boldsymbol{\kappa}$ is extinction coefficient, $\lambda$ is the wavelength in vacuum, $n$ is the index of refraction of IR absorption layer, $\alpha^{-1}$ is the penetration depth corresponding that IR intensity is reduced to $e^{-1}$ times of the intensity $I_{0}(1-R)$. If the depth is shown by $d_{\text {penetration }}[10]$, therefore,

$$
d_{\text {penetration }}=\frac{\lambda}{4 \pi n \kappa}
$$

The extinction coefficient of SiNx with higher IR absorption coefficient, lower coefficient of thermal expansion and stress by LPCVD is approximately equal to 1 . For IR radiation of the wavelength of $8 \sim 14 \mu \mathrm{m}$, according to Eq. (4), the penetration depth is about $0.3 \sim 0.4 \mu \mathrm{m}$. When the IR radiation inflects into the side of SiNx material of a bi-material beam, some of the IR radiation energy is absorbed, the other not to be absorbed will reach the metal film. As the reflectivity of Au to IR radiation is $98.4 \%$ (table 1 ), so most of radiation energy reaching the metal film will be reflected back $[11,12]$ to SiNx layer and will be absorbed by SiNx again. According to the above conclusion that the thinner the thickness of SiNx and Au film, the higher the thermal-mechanical response of beam will be, therefore, the thickness of SiNx film is selected as $0.4 \mu \mathrm{m}$. This thickness is able to both absorb $90 \%$ IR radiation energy of $8 \sim 14 \mu \mathrm{m}$ wavelength at least and ensure the value of $\Delta \theta / \Delta T$ large enough.

Above analysis indicates that, although the thinner the thickness of SiNx, the higher the thermo-mechanical response will be, SiNx absorbing IR radiation from objects should have a certain thickness to ensure a sufficient amount of radiation energy absorbed. In addition, too thin SiNx layer will make the pixel too sensitive to vibration, increased the mechanical noise of the pixel; it will also reduce the mechanical strength of the FPA, making the chip processing difficult or impossible.

\subsection{Film thickness matching}

For AulCrlSiNx structure, if thickness of $\mathrm{Cr}$ is designed as $5 \mathrm{~nm}$, the isolines of $\Delta \theta / \Delta T$ are shown as fig. 8 by eq. (1). With the aid of analyzing the isolines, the following conclusion is written as:

When the thickness of SiNx is selected, the maximum $\Delta \theta / \Delta T$ value corresponds to a certain thickness of $A u$. It is said that the maximum thermo-mechanical response of the bi-material beam may be obtained by matching the thickness of the films used for the pixel.

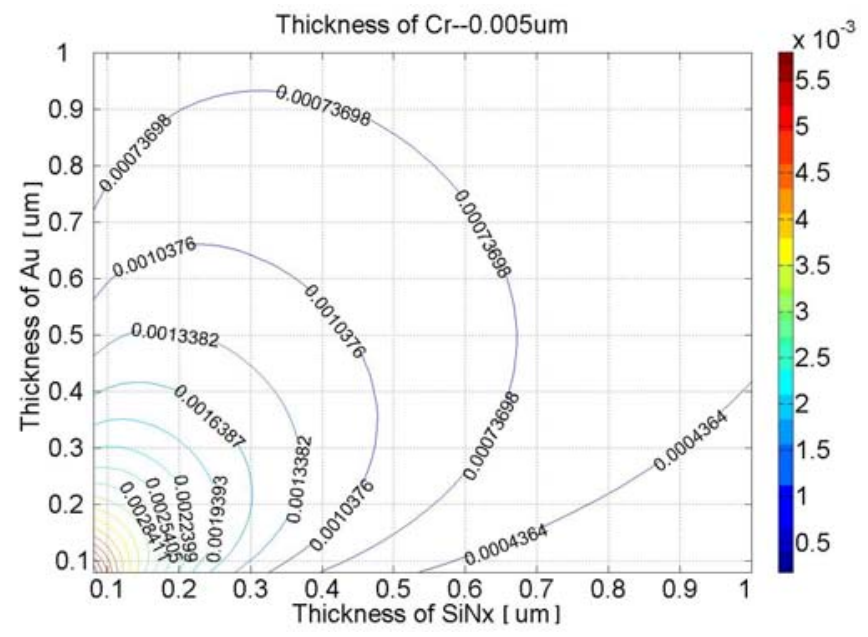

Fig. 8. Isolines of $\Delta \theta / \Delta T$

\subsection{Summary}

While thickness of two metal films on SiNx film approaches zero, the value of $\Delta \theta / \Delta T$ is close to zero also, this provides a guideline for the design of the reflector in the pixel. Regardless of the metal with smaller CET lies in the middle or the surface layer, the thinner the metal film thickness, the larger the obtained value of $\Delta \theta / \Delta T$ of the beam, which provides a guideline for the design of adhesive metal thickness. The thinner the thickness of SiNx and two metal 
films on it, the larger the value of $\Delta \theta / \Delta T$ of the beam will be, which provides a guideline for the design of high thermomechanical response. And the maximum thermo-mechanical response of the bi-material beam may be obtained by matching film thicknesses in the pixel. In addition, the thickness of the reflection surface metal is larger than 40nm, ensuring sufficient reflectivity to visible light and the SiNx thickness selected as $0.4 \mu \mathrm{m}$ can absorb $90 \%$ IR radiation energy of $8 \sim 10 \mu \mathrm{m}$ in wavelength at least.

\section{AulCrlSiNx pixel design and simulation}

While the pixel operates, it is hoped that the bi-material beams have high thermo-mechanical response and reflectors remain flat. In order to obtain high thermo-mechanical response, the SiNx thickness $h_{1}$ is selected as $0.4 \mu \mathrm{m}$, an adhesive metal layer $h_{2}$ is selected as $5 \mathrm{~nm}$. If the length of beam is $104 \mu \mathrm{m}$, a result can be acquired by Eq. (1) that the maximum value of $\Delta \theta / \Delta T$ is $2.573 \times 10^{-3} \mathrm{rad} / \mathrm{K}$, while the thickness of $A u$ is $0.29 \mu \mathrm{m}$. In order to make the reflectors flat, the Au thickness of the reflection surface is selected as $40 \mathrm{~nm}$.

Taking an example of AulCrISiNx pixel structure[2], assuming that the bi-material beam is $2 \mu \mathrm{m}$ in width and 104 $\mu \mathrm{m}$ in equivalent length, $\mathrm{Au}, \mathrm{Cr}$ and $\mathrm{SiNx}$ thicknesses are selected as $0.29 \mu \mathrm{m}, 5 \mathrm{~nm}$ and $0.4 \mu \mathrm{m}$ respectively. Applying to ANSYS software, using the model of multi-layer shell finite element, a simulating result is given that the rotation angle of the reflector is $2.614 \times 10^{-3} \mathrm{rad} / \mathrm{K}$ under one degree change of temperature, as shown in Figure 9 . Calculated values by Eq. (1) compared with simulation results; the relative error is of only $1.67 \%$ 。

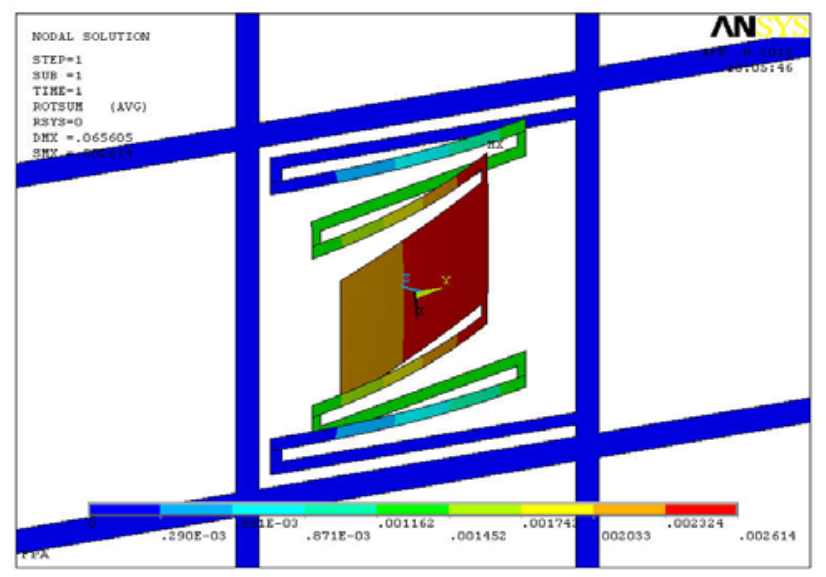

Fig. 9. An A/lCrISiNx pixel simulation result

\section{Conclusion}

Through analyzing and selecting the thickness of infrared absorption layer, visible light reflection layer and adhesive layer, and matching the thickness of various layers in the pixel, the pixel with optimized performance is obtained. For the optimized UOR IR FPA pixel, when a bi-material beam is $2 \mu \mathrm{m}$ in width and $104 \mu \mathrm{m}$ in equivalent length, the thicknesses of SiNx, Cr and Au layer are $0.4 \mu \mathrm{m}, 5 \mathrm{~nm}$, and $0.29 \mu \mathrm{m}$ respectively, the thermo-mechanical response of the designed pixel is $2.573 \times 10^{-3} \mathrm{rad} / \mathrm{K}$ calculated by Eq. (1), verified by ANSYS simulation.

\section{REFERENCES}

[1] Xia Zhang., Binbin Jiao, Dapeng Chen, Tianchun Ye, "The optimizing designing of bi-material micro cantilever with adhesive layer in between and its application in an uncooled MEMS IR FPA". Proc. SPIE, Vol. 7383, 73834M, 2009.

[2] Binbin Jiao, Chaobo, Li, Dapeng Chen et al., "A Novel Opto-mechanical Uncooled Infrared Detector". Infrared Physics \& Technology, Vol. 51(1), pp. 66-72, 2007.

[3] Yang Zhao, "Optomechanical uncooled infrared imaging system". Dissertation of UC, Berkeley, pp. 67, 2002.

[4] Freund L. B., Sureshizhu S., "Think film materials: stress, defect formation, and surface evolution". pp.212213, Science Press, Beijing, 2007,

[5] Zongfan Wu, Meilin, Liu, and Shaoju, Zhang, "Infrared and Weak Light Technology]". pp. 76, National Defense Industry Publish House, Beijing, 1998.

[6] Tairan Hsu, "MEMS \& Microsystem: Design and Manufacture". pp. 225, China Machine Industry Press, Beijing, 2004. 
[7] Freund L. B., Sureshizhu S., "Think film materials: stress, defect formation, and surface evolution". pp. 8182, Science Press, Beijing, 2007.

[8] Jun Chen. "Electromagnetic Theory of Optics". Science Press, Beijing,

[9] Sze, S. M., Ng, K work K., "Physics of Semiconductor Devices (Third Edition)", pp.41, Xian Jiao Tong University Press, Xian, 2008.

[10] Kaihua Zhao. "Optics". pp. 230 Peking University Press, Beijing, 1984.

[11] Shousheng Yan. "Foundation of Solid State Physics (Second Edition)". pp. 17, Peking University Press, Beijing, 2003.

[12] Kittel C., "Introduction of Solid State Physics (Eighth Edition)". pp. 308, Chemistry Industry Publish House, Beijing, 2005. 